\title{
Revealing evolutionary pathways by fitness landscape reconstruction
}

\author{
Manjunatha Kogenaru, Marjon G. J. de Vos , and Sander J. Tans \\ FOM Institute AMOLF, Amsterdam, The Netherlands
}

\begin{abstract}
The concept of epistasis has since long been used to denote non-additive fitness effects of genetic changes and has played a central role in understanding the evolution of biological systems. Owing to an array of novel experimental methodologies, it has become possible to experimentally determine epistatic interactions as well as more elaborate genotype-fitness maps. These data have opened up the investigation of a host of long-standing questions in evolutionary biology, such as the ruggedness of fitness landscapes and the accessibility of mutational trajectories, the evolution of sex, and the origin of robustness and modularity. Here we review this recent and timely marriage between systems biology and evolutionary biology, which holds the promise to understand evolutionary dynamics in a more mechanistic and predictive manner.
\end{abstract}

Keywords: Epistasis; fitness landscape; robustness;regulatory networks; evolution

\section{Introduction}

Genomic sequencing has generated a wealth of information on the molecular basis of organisms and their evolutionary relationships (Benson et al., 2004; Otto, 1997). However, we remain largely ignorant about the interactions between genes that are central to organismal functions and phenotype (Blattner et al., 1997). Information on how phenotypes depend on these interactions is not only relevant for understanding the architecture of cellular functions (Schuldiner et al., 2005; Collins et al., 2006; 2007), but also has profound implications for their evolutionary origin (Lunzer et al., 2005; Zhu et al., 2005; Miller et al., 2006; Dean and Thornton, 2007).

The concept of epistasis provides an elementary description of genetic interactions that are involved in function or fitness (Wright, 1932; Kauffman and Levin, 1987; Arnold et al., 2001). About 100 years ago, William Bateson introduced this term to describe phenotypic deviations from Mendelian segregation ratios due to genes masking the effects of others (Bateson, 1907). Broadly defined, epistasis denotes cases in which the effect of a mutation depends on the genetic background in which it occurs (Poelwijk et al., 2007). For instance, a mutation that is beneficial in one genetic background can be neutral or deleterious in another. Epistasis has played a central role in many evolutionary theories, including those that address speciation (Orr and Turelli, 2001), the evolution of sex (Kondrashov, 1988; Kondrashov and Kondrashov, 2001; Kouyos et al., 2006; de Visser and Elena, 2007; Gandon and Otto, 2007) and adaptation (Wright, 1931; Masel, 2005; Martin et al., 2007; Poelwijk et al., 2007).

Owing to novel experimental approaches, epistatic interactions can now be studied in an increasingly systematic manner. These efforts address a diverse array of biological systems and scientific questions. Some have a predominantly functional perspective, while others are motivated by evolutionary questions. In some cases the focus is on epistatic interactions within a single gene (intra-genic epistasis), while other studies consider larger networks of interacting genes (inter-genic epistasis). The aim of this review is to sketch these recent developments, by giving a number of illustrative examples of these diverse directions. In doing so, we hope to provide an

Address for Correspondence: Sander J. Tans, FOM Institute AMOLF, Science Park 113, 1098 XG, Amsterdam, The Netherlands. E-mail: tans@amolf.nl

(Received 01 April 2009; revised 12 May 2009; accepted 13 May 2009) 
overview of the current possibilities and limitations, and to identify new questions within this exciting new field.

\section{Intra-genic epistasis}

The nature of the epistatic interactions within a biological system is intrinsically linked to its evolutionary origin and potential. For instance in the absence of epistatic interactions between loci, genetic changes at these loci contribute independently to fitness (Figure 1A). Note
A
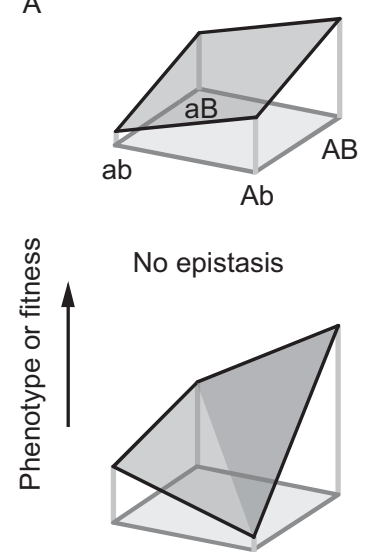

Sign epistasis

Genotype

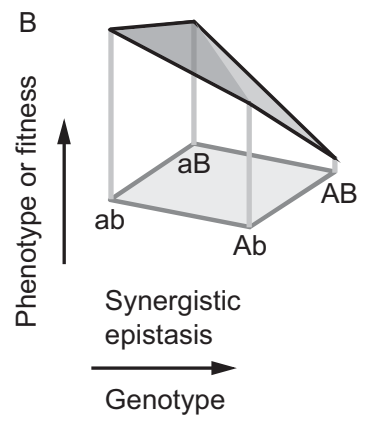

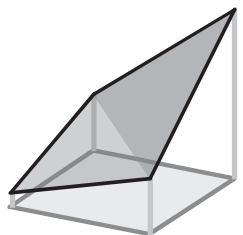

Magnitude epistasis

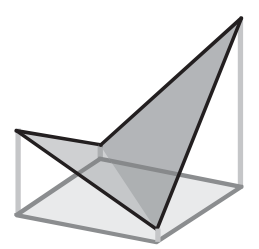

Reciprocal sign epistasis

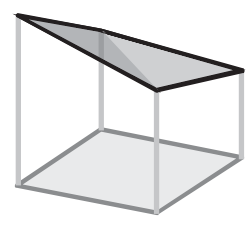

Antagonistic epistasis
Figure 1. Two classifications of epistatic interactions. A. Paths composed of two mutations are considered, from an initial sequence "ab" towards the optimum sequence "AB". When there is no epistasis, mutation " $\mathrm{a}$ " to " $\mathrm{A}$ " yields the same fitness effect for different genetic backgrounds (" $b$ " or " $\mathrm{B}$ "), while for magnitude epistasis the fitness effect may differ in magnitude, but not in sign. For sign epistasis, the sign of the fitness effect changes. Consequently, some paths become inaccessible. Finally, such a change in sign of the fitness effect can occur for both mutations; this is denoted as reciprocal sign epistasis, and is required for having multiple peaks in the fitness landscape. B. Two types of epistasis that distinguish possible interactions between two genes. Paths are considered from the initial optimal sequence "ab" towards the double knockouts "AB". If the fitness effect of the double knockout is larger than expected from the sum of their individual effects is denoted as synergistic epistasis, while a smaller than additive effect is termed as antagonistic epistasis. that for a Malthusian fitness parameter such as the bacterial growth rate, independence implies that fitness effects are additive, while when fitness is defined as the number of offspring, the individual contributions to fitness multiply. Starting from a sub-optimal phenotype, all adaptive trajectories towards the optimum then rise monotonically in fitness. Consequently, these trajectories are all equally probable to be followed during adaptation. In contrast, when required genetic changes exhibit sign-epistatic interactions (Figure 1A), some trajectories contain fitness decreasing steps, making them much less probable, though other trajectories do exhibit monotonously increasing fitness. We note that any type of epistatic interaction (not only sign epistasis) will result in some difference in the probabilities for different paths. Fitness landscapes are an intuitive concept to consider multiple possible trajectories between two points, and in both previous cases the landscape is smooth and singlepeaked. However, landscapes can also be rugged and have more than a single fitness peak. Adapting from one peak to the other then requires two or more simultaneous genetic changes, which is denoted as reciprocal sign epistasis (Figure 1A).

What are the shapes of actual fitness landscapes? This longstanding question is now starting to be addressed. Weinreich and colleagues focused on the protein $\beta$-lactamase in Escherichia coli. One variant was known to confer resistance to penicillin, while adding five mutations conferred resistance to the newer antibiotic cefotaxime. To gain insight in all possible mutational trajectories between these two variants, all $2^{5}=32$ possible intermediates were constructed and assayed on survival ability in cefotaxime, which is taken as a measure of fitness. The data revealed that a majority of the trajectories contained fitness decreasing or neutral steps, resulting in much reduced chances of being followed by natural selection (Weinreich et al., 2006).

Sign-epistatic interactions underlie these landscape features. For example, Gly238Ser in wild-type background increases the resistance, even though it increases protein aggregation by lowering the thermodynamic stability. This loss of stability is rescued by Met182Thr which alone modestly reduces resistance. Paths that fix Gly238Ser before Met182Thr are therefore plausible, but the reverse order is not. Such a balancing between functional and structural benefits is a more general evolutionary mechanism (DePristo et al., 2005; Bloom et al., 2006) and provides a mechanistic rationale for sign epistasis (Poelwijk et al., 2007; Dean and Thornton, 2007).

One may consider epistasis in fitness or at the functional level. The relation between the two was investigated using isopropylmalate dehydrogenase (IMDH) as a model system. IMDH is involved in biosynthesis of the amino acid leucine, and uses the coenzyme 
nicotinamide adenine dinucleotide $\left(\mathrm{NAD}^{+}\right)$as a hydride acceptor during an oxidative decarboxylation. Upon six mutations IMDH exchanges this coenzyme for another, nicotinamide adenine dinucleotide phosphate $\left(\mathrm{NADP}^{+}\right)$which is also used by a highly divergent paralog isocitrate dehydrogenase (IDH) (Zhu et al., 2005). The construction of a large number of IMDH intermediates, and analysis of their enzymatic activity in vitro, showed a lack of epistasis: all investigated mutations contributed roughly additively to the enzymatic activity. Thus, the genotype-phenotype relation for IMDH coenzyme use is a single featureless peak (Lunzer et al., 2005).

Assays of the same mutants in vivo, in which the corresponding growth rates were measured, yielded insight into the relation between genotype and fitness. Within this landscape, many - but not all - mutational trajectories from $\mathrm{NADP}^{+}$to $\mathrm{NAD}^{+}$usage exhibited a fitness dip, which indicates sign epistasis. This introduction of epistasis can be understood from the nonlinear relation between enzymatic activity and growth rate, in combination with the competition between two coenzymes: the fitness decreases when the recognition of $\mathrm{NADP}^{+}$is broken down, and rises again when $\mathrm{NAD}^{+}$recognition is built up. However, because apparently some mutations exist that simultaneously decrease $\mathrm{NADP}^{+}$ interaction and increase $\mathrm{NAD}^{+}$interaction, monotonously increasing trajectories are also possible. Thus although the genotype-phenotype map that depends on the functional activity of an enzyme can be free of epistasis, the corresponding phenotype-fitness map can contain (sign) epistatic features.

\section{Inter-genic epistasis}

Epistatic interactions can occur between different genetic components when they are functionally related within a network. An elementary example is the recognition between a transcription factor and its binding site within a regulatory region. Which types of epistasis underlie such a molecular recognition and which landscape features they present, was investigated using the lac repressor and operator as a model system (Poelwijk et al., 2006). Earlier mutational analysis had shown that two repressor residues and four base-pairs in the operator were central to altering the specificity of binding (Lehming et al., 1990). A large set of mutants with substitutions at these loci had been assayed on their repression value, the ratio between repressed and unrepressed expression of the controlled gene. This genotype-phenotype relation exhibited several distinct peaks: optimal repressor-operator combinations whose paths between them contained significant decreases in repression (Poelwijk et al., 2006). The existence of multiple peaks cannot be explained by sign epistasis alone, but requires the more severe reciprocal sign epistasis (Figure 1A). An intuitive rationale for this type of epistasis is the following: mutating one binding partner likely only benefits a new interaction if the other binding partner is mutated first and vice versa. The alternative outcome, in which optima are bridged by a "master key" repressor that binds to multiple operators, was not observed in the data.

As the repressor-operator binding landscape contains many sub-optima, they would seem to act as evolutionary traps that hamper adaptation to the global optima. One phenomenon that may affect this outcome is the presence of duplicated genes and their mutational divergence. E. coli contains several homologs of the lac repressor, which regulate the expression of different operons independently and must therefore have evolved specific repressor-operator recognition. Indeed, the promiscuous binding of functionally unrelated transcription factors on the lac operator should provide a selective pressure on binding specificity. This issue was investigated using the lac genotype-repression landscape. It was found that for two identical repressoroperator pairs, many mutational trajectories now did not exhibit decrease in fitness. Mutations appeared to exist for which the obligatory decrease in repression for one repressor was offset by a simultaneous decrease in the penalty for promiscuous binding of the other repressor. Such compensations within biochemical networks are ubiquitously observed and may therefore be a more general adaptive evolution mechanism (Francino, 2005). These results also substantiate the suggestion that the robustness of networks may promote evolvability (Conant and Wagner, 2003; Lynch, 2005).

Within the larger web of biological interactions, epistasis originates not only by direct physical recognition, but also through their hidden functional relations. Elena and colleagues explored this issue by creating random single and double mutants in $E$. coli, using Tn10 transposition and P1vir transduction (Elena and Lenski, 1997). Epistasis is absent if the fitness effect of the double mutant equals the sum of the fitness effects of the single mutants. However, a larger than additive effect of the double mutant is denoted as synergistic epistasis (Figure 1B), while a smaller than additive effect indicates antagonistic epistasis (Figure 1B). The data showed that epistasis between these random knockouts is not rare, and that antagonistic and synergistic interactions occur almost at the same frequency (Elena and Lenski, 1997; Otto, 1997). This observation is also supported by experimental studies from other organisms (Burch et al., 2003; Elena, 1999; Wloch et al., 2001; de Visser, 1997; Sanjuan et al., 2004; Rowe et al., 2008) and by computational modeling (Lenski et al., 1999; You and Yin, 2002). 
The conservation of epistatic interactions across different organisms was addressed by Tischler and colleagues. This study focused on synthetic lethal interactions, a phenomenon in which two non-lethal mutations yield a lethal phenotype when combined. A large number of synthetic lethal interactions between genes are known for Saccharomyces cerevisiae, some of which have orthologous pairs in Caenorhabditis elegans. These 837 pairs were assayed in C. elegans on fitness using RNA interference (RNAi). It was found that a maximum of $5 \%$ of synthetic lethal interactions are conserved between S. cerevisiae and C. elegans. This value is low compared to the conservation of proteinprotein interactions (31\%) (Tischler et al., 2008). Thus, surprisingly, even though the gene function between worm and yeast are conserved, the epistatic interactions are not.

The aspect common to the above studies is that they assess epistasis through measurement of fitness. As the molecular mechanisms that underlie fitness are often poorly understood, inference of epistasis from fitness alone usually does not provide a mechanistic explanation of epistasis. Epistasis can also be detected at lower levels of organization, such as transcription, for which the underlying mechanisms are better understood. One study focused on two glucose-adapted lines of E. coli (Cooper et al., 2008) and the ancestral line, as well as crp knockouts for each of these lines. crp is a key global regulatory gene that itself was not altered in the glucose adaptation (Cooper et al., 2003). Deletion of the crp gene appeared more detrimental to growth in the evolved strains compared to the ancestor. The cause of this effect was found in the adapted expression profile: in the ancestral strain, crp controls the expression of 171 genes whereas in the evolved strains it controls an additional 115 genes that were not initially crp-dependent (Gosset et al., 2004). This study highlights the importance of lower-level phenotypic characterization when aiming to understand the mechanisms that underlie epistasis.

Epistasis is also thought to be related to the occurrence of sexual recombination. In the 1980s, Kondrashov introduced the mutational deterministic hypothesis, which states that the deleterious mutations that are combined during recombination are purged from the population (Kondrashov, 1988). This scenario requires synergistic epistasis, as it makes combined mutations more harmful than expected from their individual effects. The relation between epistasis and sexual reproduction has been investigated in a computational study of networks of transcriptional regulators as found in Drosophila melanogaster (Jeager et al., 2004; Azevedo et al., 2006). Individuals who attained stable expression patterns upon variation of their network interactions and selection were considered viable, whereas individuals that failed to reach equilibrium were considered not viable. The simulations showed that synergistic epistasis, and accordingly mutational robustness, indeed increased within the network in a sexually reproducing population while it did not in asexual populations. This effect is more prominent especially if the population experiences a high mutation rate and there are many genetic interactions. It remains to be seen whether these results in turn also provide a rationale for the maintenance of sex despite its costs.

The relation between epistasis and modularity was addressed in a computational study of $S$ cerevisiae metabolism. By deleting single and pairs of genes in silico and computing the resulting growth rate using flux balance analysis, epistatic interactions could be assessed. Between two functional modules, such as those responsible for respiration and glycolysis, significant epistatic interactions were observed. These epistatic interactions were either consistently synergistic or consistently antagonistic. On the other hand, within a functional module both types of epistasis were observed. These results point to a correlation between network architecture and epistasis, and challenge the common notion that epistasis is stronger within a functional module than between the modules (Moore, 2005; Segre et al., 2005).

In addition to epistatic interactions between genotypes, interactions between genotype and environment are also common. In this case, the effect of a mutation depends on the environment in which fitness is assayed and vice versa. To investigate genotype by environment interactions, random insertion mutants of $E$. coli were assayed in four different environments. It was found that about $40 \%$ of the insertions yielded different fitness effects in the different environments, showing that genotype by environment interactions are common (Remold and Lenski, 2001).

\section{Conclusions and perspective}

The studies reviewed here illustrate that epistasis plays a central role in a broad array of systems, scientific questions and experimental methodologies. The questions range from being predominantly functional, where epistasis is a powerful tool to unravel functional relations between genes, to mainly evolutionary questions, where epistasis provides a mechanistic explanation or even a prediction of adaptive dynamics. Some research focuses on interactions between residues within a single protein, while others consider the full regulatory networks underlying organismal fitness. These diverse approaches have provided an intriguing insight into hidden correlations within the design and evolutionary potential of biological systems. 
However, the current approaches have considerable limitations and only begin to scratch the surface of all relevant correlations. For instance, intra-genic studies have so far only explored base-pair substitutions within restricted parts of genotype space. One may argue that organisms can exploit genetic changes in other regions to achieve the same functionality, thus making observed constraint and epistasis irrelevant. However, phylogenetic analysis shows that evolutionary transitions similar to several studied examples have in fact occurred in evolutionary history. Larger scale genomic rearrangements such as recombination are also known to play an essential role in the evolution of some functions. It would be of interest to explore how recombination events as well as ploidity affect fitness landscapes and the evolutionary trajectories within it. On the other hand, studies of epistasis within larger networks do explore more distant interactions, but in return typically only consider knockouts as the genetic change, and lethality as the response. It would be fruitful to investigate more detailed changes, such as altered expression levels or point mutations resulting in novel functionality, as well as more quantitative fitness measurements.

The complexity of biological and ecological systems makes finding new questions easy. For instance, most fitness landscapes and epistases studied so far have considered straightforward constant environments, even though much of the evolutionary innovation in nature likely results from adaptation in variable environments. The fitness landscape and epistasis concepts hold promise to also address the role of environmental variability in a more mechanistic manner. One may also wonder about the causal relation between epistasis and structure or function. Is biological function and structure predominantly shaped by epistasis, or vice versa, are epistatic interactions shaped by structure and function?

Recent decades have brought detailed functional insight at the molecular level. Increased understanding at the network and systemic level now enables a link to fitness, and allows one to address evolutionary dynamics throughout all levels of biological organization in a quantitative and mechanistic manner.

\section{Acknowledgements}

This work is part of the research programme of the Stichting voor Fundamenteel Onderzoek der Materie (FOM), which is financially supported by the Nederlandse Organisatie voor Wetenschappelijke Onderzoek (NWO).

Declaration of interest: The authors report no conflict of interest.

\section{References}

Arnold SJ, Pfrender ME and Jones AG. 2001. The adaptive landscape as a conceptual bridge between micro- and macroevolution. Genetica 112-113:9-32.

Azevedo RB, Lohaus R, Srinivasan S, Dang KK and Burch CL. 2006. Sexual reproduction selects for robustness and negative epistasis in artificial gene networks. Nature 440:87-90.

Bateson W. 1907. Facts limiting the theory of heredity. Science 26:649-660.

Benson DA, Karsch-Mizrachi I, Lipman DJ, Ostell J and Wheeler DL. 2004. GenBank: update. Nucleic Acids Res 32:D23-26.

Blattner FR, Plunkett G 3rd, Bloch CA, Perna NT, Burland V, Riley M, Collado-Vides J, Glasner JD, Rode CK, Mayhew GF, Gregor J, Davis NW, Kirkpatrick HA, Goeden MA, Rose DJ, Mau B, Shao Y. 1997. The complete genome sequence of Escherichia coli K-12. Science 277:1453-1474.

Bloom JD, Labthavikul ST, Otey CR and Arnold FH. 2006. Protein stability promotes evolvability. Proc Natl Acad Sci USA 103:5869-5874.

Burch CL, Turner PE and Hanley KA. 2003. Patterns of epistasis in RNA viruses: a review of the evidence from vaccine design. J Evol Biol 16:1223-1235.

Collins SR, Schuldiner M, Krogan NJ and Weissman JS. 2006. A strategy for extracting and analyzing large-scale quantitative epistatic interaction data. Genome Biol 7:R63.

Collins SR, Miller KM, Maas NL, Roguev A, Fillingham J, Chu CS, Schuldiner M, Gebbia M, Recht J, Shales M, Ding H, Xu H, Han J, Ingvarsdottir K, Cheng B, Andrews B, Boone C, Berger SL, Hieter P, Zhang Z, Brown GW, Ingles CJ, Emili A, Allis CD, Toczyski DP, Weissman JS, Greenblatt JF, Krogan NJ. 2007. Functional dissection of protein complexes involved in yeast chromosome biology using a genetic interaction map. Nature 446:806-810.

Conant GC and Wagner A. 2003. Asymmetric sequence divergence of duplicate genes. Genome Res 13:2052-2058.

Cooper TF, Rozen DE and Lenski RE. 2003. Parallel changes in gene expression after 20,000 generations of evolution in Escherichia coli. Proc Natl Acad Sci USA 100:1072-1077.

Cooper TF, Remold SK, Lenski RE and Schneider D. 2008. Expression profiles reveal parallel evolution of epistatic interactions involving the CRP regulon in Escherichia coli. PLoS Genet 4:e35.

Dean AM and Thornton JW. 2007. Mechanistic approaches to the study of evolution: the functional synthesis. Nat Rev Genet 8:675-688.

DePristo MA, Weinreich DM and Hartl DL. 2005. Missense meanderings in sequence space: a biophysical view of protein evolution. Nat Rev Genet 6:678-687.

de Visser JA and Elena SF. 2007. The evolution of sex: empirical insights into the roles of epistasis and drift. Nat Rev Genet 8:139-149.

de Visser JAGM, Hoekstra RF and van den Ende H. 1997. Test of interaction between genetic markers that affect fitness in Aspergillus niger. Evolution 51:1499-1405.

Elena SF. 1999. Little evidence for synergism among deleterious mutations in a nonsegmented RNA virus. J Mol Evol 49:703-707.

Elena SF and Lenski RE. 1997. Test of synergistic interactions among deleterious mutations in bacteria. Nature 390:395-398.

Francino MP. 2005. An adaptive radiation model for the origin of new gene functions. Nat Genet 37:573-577.

Gandon S and Otto SP. 2007. The evolution of sex and recombination in response to abiotic or coevolutionary fluctuations in epistasis. Genetics 175:1835-1853.

Gosset G, Zhang Z, Nayyar S, Cuevas WA and Saier Jr MH. 2004. Transcriptome analysis of Crp-dependent catabolite control of gene expression in Escherichia coli. J Bacteriol 186:3516-3524.

Jaeger J, Blagov M, Kosman D, Kozlov KN, Manu, Myasnikova E, Surkova S, Vanario-Alonso CE, Samsonova M, Sharp DH, Reinitz J. 2004. Dynamical analysis of regulatory interactions in the gap gene system of Drosophila melanogaster. Genetics 167:1721-1737. 
Kauffman S and Levin S. 1987. Towards a general theory of adaptive walks on rugged landscapes. J Theor Biol 128:11-45.

Kondrashov AS. 1988. Deleterious mutations and the evolution of sexual reproduction. Nature 336:435-440.

Kondrashov FA and Kondrashov AS. 2001. Multidimensional epistasis and the disadvantage of sex. Proc Natl Acad Sci USA 98:12089-12092.

Kouyos RD, Otto SP and Bonhoeffer S. 2006. Effect of varying epistasis on the evolution of recombination. Genetics 173:589-597.

Lehming N, Sartorius J, Kisters-Woike B, von Wilcken-Bergmann B and Muller-Hill B. 1990. Mutant lac repressors with new specificities hint at rules for protein-DNA recognition. Embo J 9:615-621

Lenski RE, Ofria C, Collier TC and Adami C. 1999. Genome complexity, robustness and genetic interactions in digital organisms. Nature 400:661-664.

Lunzer M, Miller SP, Felsheim R and Dean AM. 2005. The biochemical architecture of an ancient adaptive landscape. Science 310:499-501

Lynch M. 2005. Simple evolutionary pathways to complex proteins. Protein Sci 14:2217-2225; discussion 2226-2227.

Martin G, Elena SF and Lenormand T. 2007. Distributions of epistasis in microbes fit predictions from a fitness landscape model. Nat Genet 39:555-560.

Masel J. 2005. Evolutionary capacitance may be favored by natural selection. Genetics 170:1359-1371.

Miller SP, Lunzer M and Dean AM. 2006. Direct demonstration of an adaptive constraint. Science 314:458-461.

Moore JH. 2005. A global view of epistasis. Nat Genet 37:13-14.

Orr HA and Turelli M. 2001. The evolution of postzygotic isolation: accumulating Dobzhansky-Muller incompatibilities. Evolution 55:1085-1094.

Otto SP. 1997. Unravelling gene interactions. Nature 390:343.

Poelwijk FJ, Kiviet DJ and Tans SJ. 2006. Evolutionary potential of a duplicated repressor-operator pair: simulating pathways using mutation data. PLoS Comput Biol 2:e58.

Poelwijk FJ, Kiviet DJ, Weinreich DM and Tans SJ. 2007. Empirical fitness landscapes reveal accessible evolutionary paths. Nature 445:383-386.
Remold SK and Lenski RE. 2001. Contribution of individual random mutations to genotype-by-environment interactions in Escherichia coli. Proc Natl Acad Sci USA 98: 11388-11393.

Rowe HC, Hansen BG, Halkier BA and Kliebenstein DJ. 2008. Biochemical networks and epistasis shape the Arabidopsis thaliana metabolome. Plant Cell 20:1199-1216.

Sanjuan R, Moya A and Elena SF. 2004. The contribution of epistasis to the architecture of fitness in an RNA virus. Proc Natl Acad Sci USA 101:15376-15379.

Schuldiner M, Collins SR, Thompson NJ, Denic V, Bhamidipati A, Punna T, Ihmels J, Andrews B, Boone C, Greenblatt JF, Weissman JS, Krogan NJ. 2005. Exploration of the function and organization of the yeast early secretory pathway through an epistatic miniarray profile. Cell 123:507-519.

Segre D, Deluna A, Church GM and Kishony R. 2005. Modular epistasis in yeast metabolism. Nat Genet 37:77-83.

Tischler J, Lehner B and Fraser AG. 2008. Evolutionary plasticity of genetic interaction networks. Nat Genet 40:390-391.

Weinreich DM, Delaney NF, Depristo MA and Hartl DL. 2006. Darwinian evolution can follow only very few mutational paths to fitter proteins. Science 312:111-114.

Wloch DM, Szafraniec K, Borts RH and Korona R. 2001. Direct estimate of the mutation rate and the distribution of fitness effects in the yeast Saccharomyces cerevisiae. Genetics 159:441-452.

Wright S. 1931. Evolution in Mendelian populations. Genetics 16:97-159.

Wright, S. 1932. The role of mutation, inbreeding, crossbreeding and selection in evolution. Proceedings of the VI International Congress of Genetics. 1: 356-366.

You L and Yin J. 2002. Dependence of epistasis on environment and mutation severity as revealed by in silico mutagenesis of phage t7. Genetics 160:1273-1281.

Zhu G, Golding GB and Dean AM. 2005. The selective cause of an ancient adaptation. Science 307:1279-1282.

Editor: Michael M. Cox 